\title{
ARTICLE \\ Diversity in global gene expression and morphology across a watercress (Nasturtium officinale R. Br.) germplasm collection: first steps to breeding
}

\author{
Adrienne C. Payne ${ }^{1}$, Graham J.J. Clarkson ${ }^{2}$, Steve Rothwell ${ }^{2}$ and Gail Taylor ${ }^{1}$
}

\begin{abstract}
Watercress (Nasturtium officinale R. Br.) is a nutrient intense, leafy crop that is consumed raw or in soups across the globe, but for which, currently no genomic resources or breeding programme exists. Promising morphological, biochemical and functional genomic variation was identified for the first time in a newly established watercress germplasm collection, consisting of 48 watercress accessions sourced from contrasting global locations. Stem length, stem diameter and anti-oxidant (AO) potential varied across the accessions. This variation was used to identify three extreme contrasting accessions for further analysis. Variation in global gene expression was investigated using an Affymetrix Arabidopsis ATH1 microarray gene chip, using the commercial control (C), an accession selected for dwarf phenotype with a high AO potential (dwarfAO, called 'Boldrewood') and one with high AO potential alone. A set of transcripts significantly differentially expressed between these three accessions, were identified, including transcripts involved in the regulation of growth and development and those involved in secondary metabolism. In particular, when differential gene expression was compared between $\mathrm{C}$ and dwarfAO, the dwarfAO was characterised by increased expression of genes encoding glucosinolates, which are known precursors of phenethyl isothiocyanate, linked to the anti-carcinogenic effects well-documented in watercress. This study provides the first analysis of natural variation across the watercress genome and has identified important underpinning information for future breeding for enhanced anti-carcinogenic properties and morphology traits in this nutrient-intense crop.
\end{abstract}

Horticulture Research (2015) 2, 15029; doi:10.1038/hortres.2015.29; Published online: 8 July 2015

\section{INTRODUCTION}

Watercress (Nasturtium officinale R. Br.) is a leafy crop consumed globally, which is highly nutrient intense ${ }^{1}$ and contains a wide range of natural, bioactive plant compounds for which there is increasing evidence for beneficial effects on human health. ${ }^{2,3}$ In particular, high concentrations of glucosinolates and their hydrolysis to isothiocyanates are now known to have a role in supressing tumour growth ${ }^{4,5}$ and in the prevention of several other diseases including cardiovascular, neurodegeneration and diabetes. ${ }^{6,7}$ At the same time, global consumption of this crop is increasing. In western diets this tends to be as a salad leaf eaten raw, whilst in eastern diets, the leaves and stems of the plant are cooked and used in soup. ${ }^{8}$ In a world where food security is a global concern and where sustainable intensification suggests that primary production must be achieved more efficiently, the availability of food plants with high nutritional value is likely to become more important in the future. ${ }^{9,10}$ Despite this, limited genetic or genomic resources have been procured for watercress and there is no active breeding programme, globally. Watercress has not, to date, been fully DNA sequenced but recent advances in next generation sequencing mean that rapid progress should now be possible. Next generation sequencing opens a whole new expanse of opportunities to examine the genome at relatively low cost even in an under studied crop such as watercress. ${ }^{11}$ However as a first step, insight into variation in traits of interest is still required. Given this, it is important to characterise wide germplasm collections phenotypically in order to tar- get NGS resources effectively, in addition to identifying germplasm for breeding.

Wild populations or germplasm collections often contain beneficial genetic variation that can be introgressed into breeding programmes, ${ }^{12}$ including for agronomic, nutritional or stress tolerance traits. Examples of introgression of such wild alleles into breeding programmes have been previously demonstrated ${ }^{13}$ including for increased drought and salinity tolerance which was achieved in tomatoes by introgression of genes from wild relatives. ${ }^{13}$ Traits such as soluble content and fruit colour have also been improved in tomatoes. ${ }^{14}$ Wheat, barley and rye have a limited genetic diversity and the success in breeding programmes of these cultivars is largely driven by the introgression of the alleles from wild relatives. ${ }^{15}$ Thus, for watercress, analysis of wild populations may identify useful material for breeding crops with improved phytonutrients, in addition to aiding the search for suitable material from which to develop genetic and genomic resources.

A cross species GeneChip, termed X species has been used for a range of species and in this study we hybridised watercress to the Arabidopsis chip for gene expression analysis. Arabidopsis was selected as like watercress it is a member of the Brassicaceae family. ${ }^{16}$ Several papers have reported using arrays designed for a model species to investigate the transcriptome of other species e.g. Hammond et $a .^{17}$ investigated Brassica oleracea on the ATH1 genechip with $98.8 \%$ of the available probe sets retained, Thlapsi caerulescens and Thlapsi arvense were hybridised to the Arabidopsis ATH1 genechip, ${ }^{18}$ banana (Musa species) was hybridised to both

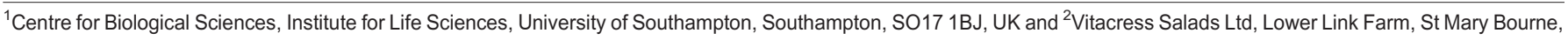
Andover, Hampshire, SP116DB, UK.

Correspondence: Gail Taylor (G.Taylor@soton.ac.uk)

Received 1 April 2015; revised 23 May 2015; accepted 26 May 2015 
the ATH1 and rice genechip ${ }^{19}$ and finally Cardamine kokaiensis hybridised to the ATH1 genechip. ${ }^{20}$

The Arabidopsis ATH1 chip contains 22,810 Arabidopsis probe sets allowing for the evaluation of almost all the genes in the Arabidopsis genome. ${ }^{21}$ The coding regions in Arabidopsis thaliana and across Brassicaceae are highly conserved with Cavell, Lydiate ${ }^{22}$ reporting an $85 \%$ sequence similarity. ${ }^{23}$ Also on a Brassica expressed sequence tag (EST) microarray made available from John Innes centre, $77 \%$ of the protein hits came from $A$. thaliana. This gave us confidence to proceed with using Arabidopsis to further watercress knowledge.

The main aim of this study was to provide underpinning data for molecular breeding by quantifying natural genetic diversity for phenotype, genotype and gene expression data (the transcriptome), in a global watercress germplasm collection held at the University of Southampton. Growth measurements were made on the collection in two contrasting environments and a broad screen for phytonutrient status was used to assess anti-oxidant (AO) potential, using FRAP. ${ }^{24}$ FRAP is a simple assay which assesses "AO potential" by ferric to ferrous ion reduction causing the formation of a ferrous-tripyridyltriazine complex which is measured using a spectrophotometer for colour change. ${ }^{25}$ Although $\mathrm{AO}$ potential is a generic measurement, it provides insight into the $A O$ potential of a wide range of phytonutrients including vitamins, phenolics, sulphur-containing compounds and glucosinolates. Our analysis provides the first assessment of functional genomic and biochemical diversity in a wild watercress collection from across several continents and as such will be important for future targeted sequencing and molecular breeding for the sustainable intensification of this nutrient-intense food crop.

This study sought to identify natural variation within a watercress germplasm collection which could be taken forward for a molecular breeding programme focused on enhancing nutritional and agronomic traits.

\section{MATERIALS AND METHODS}

\section{Growth and morphology analysis}

Watercress germplasm collection and growing conditions. Seeds were collected from commercial growers, from a variety of locations and donated from HRI, Warwick. Seeds were germinated in Petri dishes lined with two layers of moistened filter paper with lids but not sealed and then individually transferred to peat ( $\mathrm{pH} 6, \mathrm{mg} /$ litre added; N-144, P-154 and K-264) in $7.5 \mathrm{~cm}$ pots. The pots were then randomised and placed in large trays which were maintained with $50 \mathrm{~mm}$ of water and checked on a regular basis. The watercress accessions were raised in a growth room with a controlled environment (CE), $25{ }^{\circ} \mathrm{C}$ day/22 ${ }^{\circ} \mathrm{C}$ night, 16 hours day, 06:00-22:00 with PAR varying between 120 and $180 \mu \mathrm{mol} \mathrm{m} \mathrm{m}^{-2} \mathrm{~s}^{-1}$ and humidity uncontrolled. After 7 weeks of growth the whole plant minus roots were snap frozen for DNA and RNA extraction. For accessions grown in the field the accessions were initially germinated in $53 \times 32 \mathrm{~cm}$ ( 32 squares by 18 squares) sowing trays kept in a polytunnel located in Spetisbury $\left(50^{\circ} 48^{\prime} 46.8^{\prime \prime} \mathrm{N}, 2^{\circ} 08^{\prime} 47.9^{\prime \prime} \mathrm{W}\right)$, Dorset, UK with continual misting and then transferred to a watercress bed which was gravel lined with free flowing spring water. The bed was divided into sections to ensure no mixing of accessions occurred.

\section{Morphological}

Stem length, diameter and leaf number index.

The length of the longest stem on each plant was recorded in centimetres and the stem diameter at $5 \mathrm{~cm}$ up the stem was recorded for each plant after 7 weeks. The number of fully expanded leaves (leaves larger than $2 \mathrm{~cm} \times$ $2 \mathrm{~cm}$ ) was recorded. The leaf number index (LNI) was then calculated by dividing the number of leaves per plant by the stem length of that plant.

\section{Statistical analysis}

Two-way ANOVA model was applied on morphological and biochemical data to determine statistical significance:

$$
Y=A+B+A B
$$

where $\mathrm{A}=$ watercress accession and $\mathrm{B}=$ growth environment.

Anti-oxidant potential: ferric reducing ability of plasma (FRAP). The FRAP method was based on that of Benzie and Strain ${ }^{25}$ but modified by Payne et al. ${ }^{24}$

\section{Genetic analysis}

DNA extraction. DNA extraction was carried out using a CTAB protcol adapted from Doyle. ${ }^{26}$

Hybridisation to $X$ species chip. DNA from the control line (C) was sent to the National Arabidopsis Stock Centre (NASC) for hybridisation to the $X$ species ATH1 genome array.

Xspecies chip analysis - creation of probe mask (.cdf) files. Oligonucleotide GeneChip probes with low/no hybridisation to watercress transcripts were removed using the gDNA probe masking strategy devised by Hammond et al. ${ }^{17}$ and subsequently used by Davey et al. ${ }^{19}$ The custom .cdf file was then loaded onto the GeneSpring software for analysis of the CEL files.

RNA extraction. RNA was extracted from three accessions with traits of interest; control (C), high AO content and high AO content with a dwarf phenotype (dwarfAO). Accessions were grown in a CE in order to eliminate any environmental effects.

RNA was extracted using the Zymo Research (ZR) kit (Zymo Research, Irvine, CA, USA) following manufacturers guidelines.

Microarray analysis and hierarchial clustering. After selection of perfectmatch probes which hybridised efficiently to the gDNA the perfect match probes were used for interpreting the GeneChip array in which RNA from Nasturtium officinale was hybridised. Examination of gene expression was conducted using the Agilent Genespring Gx Analysis Workbench version 7.3.

Microarray analysis was performed according to the Agilent GeneSpring GX version 7.3 analysis workbench manual and Mapman 3.1 instruction manual. Microarray analysis allows the expression of thousands of genes to be investigated simultaneously. ${ }^{27}$ The .cdf file (filtered from the raw cel.file) created from the $X$ species analysis was used for the gene expression analysis. Robust multiarray averaging (RMA) normalisation was used. Statistical analysis to identify differentially expressed genes was performed with ANOVA using a $p$ cut-off of 0.05. Multi-experiment Viewer (TMeV) was used to perform hierarchical clustering, using the standard Pearsons and a distance of 0.28 .

CDNA synthesis and real-time PCR. CDNA synthesis was performed with the ImPromll Reverse Transcription kit, Promega (Southampton, UK) and manufacturers guidelines followed. Real-time PCR was performed using At1g65060 (encodes an isoform of 4-coumarate:CoA ligase (4CL) and At4g35000 (encodes a microsomal ascorbate peroxidase APX3). A 1/4 dilution of the CDNA for each sample was used, along with the primer mix and 2X SYBR master mix (Finnzymes, Thermo Scientific, Loughborough, UK). Chromo4 real-time PCR machine (Biorad) was used with the following programme: $95^{\circ} \mathrm{C}$ for 10 minutes, $95{ }^{\circ} \mathrm{C}$ for 10 seconds, $60{ }^{\circ} \mathrm{C}$ for 15 seconds, $72{ }^{\circ} \mathrm{C}$ for 15 seconds, $75{ }^{\circ} \mathrm{C}$ for 1 second, plate read, repeat from the second step 39 more times, $72{ }^{\circ} \mathrm{C}$ for 5 minutes, melting curve from $55^{\circ} \mathrm{C}$ to $95^{\circ} \mathrm{C}$, read every $0.2^{\circ} \mathrm{C}$, hold for 1 second and finally $75{ }^{\circ} \mathrm{C}$ for 5 minutes. Gene expression calculations to confirm microarray results were performed using the methods of Pfaffl. ${ }^{28}$

Mapman analysis. Mapman is a user-driven tool which displays large data sets and displays gene expression experiments on diagrams of metabolic pathways or other processes. Gene expression data were obtained from microarray analysis and the following calculation performed in order to determine differences in expression which were illustrated in Mapman.

Expression $=\log$ (gene expression of accession of interest/gene expression of control accession)

\section{RESULTS}

Morphological and biochemical variation in a watercress germplasm collection

AO potential from FRAP differed significantly across the watercress accessions when grown in a CE and also when grown in the field 
(Figure 1a). There was a dramatic increase in AO potential for field grown as compared to CE-grown watercress where the control accession increased from $130 \mathrm{mmol} \mathrm{Fe}^{2+}$ equivalent per gram fresh weight in a CE to $529 \mathrm{mmol} \mathrm{Fe}{ }^{2+}$ equivalent per gram fresh weight in the field. There was a significant difference between the watercress accessions, environment and an interaction between accession and environment (Table 1). The different watercress accessions did not rank the same in the CE and field and Spearman's rho revealed a non-significant relationship, $r_{s}(34)=$ $0.019, p=0.915$.

Stem length differed significantly across the watercress accessions irrespective of growth environment and varied by $74 \%$ and $44 \%$ in CE and field respectively (Figure 1b). When the CE grown watercress was compared to the field there was a distinct difference. The CE grown accessions had a longer stems compared to field grown accessions. There was a significant difference between the watercress accessions, environment and an interaction between accession and environment (Table 1). The different watercress accessions did not rank the same in the CE and field and Spearman's rho revealed a statistically non-significant relationship, $r_{s}(58)=0.075, p=0.569$.

Stem diameter also differed significantly across the watercress accession lines in both CE and field (Figure 1c). The mean stem diameter was generally higher for CE grown watercress compared to accessions grown in the field. Stem diameter differed significantly between watercress accessions but did not significantly differ between environments however there was a significant interaction between accession and environment (Table 1). The different watercress accessions do not rank the same in the CE and field and Spearman's rho revealed a statistically non-significant relationship, $r_{s}(58)=-0.179, p=0.171$

LNI was calculated as leaf number per unit stem length. LNI was highest for field grown watercress, possibly indicating a response to competition where swards of watercress were grown together (Figure 1d). LNI differed significantly between watercress accessions but did not significantly differ between environments however there was a significant interaction between accession and environment (Table 1). The different watercress accessions do not rank the same in the CE and field and Spearman's rho revealed a statistically non-significant relationship, $r_{s}(58)=-0.005, p=0.969$.

In summary, phenotypic and biochemical measurements revealed significant variation between the watercress accessions which led to an examination of gene expression.

Assessing global gene expression in a watercress germplasm collection

$X$ species analysis ${ }^{17,19}$ Using .cdf masking the hybridisation to the Arabidopsis ATH-1 gene chip was very high with a minimum intensity being set at 16000 in order to obtain no probe sets or probe pairs being retained. In order to determine the threshold intensity only the data from 0 to 2000 were plotted so the greatest distance between the probe-set and probe-pair could be identified. It appears that the threshold intensity was 80 . Investigation into any variation in gene expression between the three selected accessions ( $C, A O$ and dwarf $A O)$ could then be explored.
Venn diagrams; visualisation of commonalities between gene lists

Figure $2 \mathrm{a}$ explores the commonalities between gene lists of significantly different expression between the three accessions of interest, i.e. how many genes in common were up- or down-regulated between these three accessions. This would give an indication of the similarity of these three accessions to each other (control C; high $\mathrm{AO} ; \mathrm{AO}$ and dwarf high $\mathrm{AO}$; dwarfAO). The up-regulation of genes was filtered on expression above 0 and the down-regulation of genes filtered on expression below 0 after $\log _{2}$ had been applied. The examination of up- and down-regulated genes was conducted on lines $C, A O$ and dwarfAO. Accession $C$ and $A O$ had an up-regulation of 80 genes in common whilst only 36 genes were up-regulated in common between accession $C$ and dwarfAO, between accession $\mathrm{AO}$ and dwarfAO 63 genes were in common. dwarfAO had the highest number of genes up-regulated with 189 genes in total whilst $A O$ and $C$ had 177 genes. Interestingly there were 0 genes in common for all three accessions (Figure 2a). It is evident that the accessions $\mathrm{C}, \mathrm{AO}$ and dwarfAO differ at the level of the transcriptome as illustrated in the gene expression output from microarray analysis in Figure 2b, dwarfAO demonstrating the most distinct difference.

Again for down-regulation accession $\mathrm{C}$ and $\mathrm{AO}$ had the highest number of genes in common down-regulated, 400 genes whilst $C$ and dwarfAO had the lowest number of genes in common downregulated, 344 genes. AO had the overall highest number of downregulated genes with a total of 497 whilst $C$ has a total of 495 genes down-regulated and dwarfAO 484 genes down-regulated. There were 310 genes in common for all three accessions (Figure 2a).

It is important to note that it appears that $C$ and dwarfAO have the fewest common genes up- and down-regulated. Interestingly there was a smaller difference between genes which are commonly up- and down-regulated between $\mathrm{AO}$ and dwarf $\mathrm{AO}$ and also $\mathrm{C}$ and $\mathrm{AO}$.

AgriGO - biological processes, cellular component and molecular function

Gene expression data were uploaded into AgriGO (Figure S1). dwarfAO tended to give the highest $Z$ score (statistical value in PAGE) in biological processes in particular developmental process and response to stimulus. Also in terms of molecular function and cellular components dwarfAO has the highest $Z$ score. It is evident that there is different control over various biological processes, molecular functions and cellular components across these three contrasting accessions.

Multi-experiment Viewer $(\mathrm{TMeV})^{29}$; gene clustering

There were dramatic differences in gene expression between the three key accessions of interest (control, high AO content and high AO content with dwarf phenotype) (Figure 2b). Gene expression from microarray analysis was confirmed by real-time PCR (Figure S2). The list of genes which changed significantly between the watercress accessions were then uploaded to $\mathrm{TMeV}^{29}$ to investigate clustering of these genes. It is evident that $\mathrm{C}$ and $\mathrm{AO}$ were more closely related with respect to gene expression than with dwarfAO

Table 1 Statistical significance of traits of interest, shading represents significant results

\begin{tabular}{llll}
\hline Trait & \multicolumn{1}{c}{ Accession } & Environment & Accession $\times$ Environment \\
\hline Stem length & $F_{(11,96)}=9.05, p<0.001$ & $F_{(1,96)}=28.16, p<0.001$ & $F_{(11,96)}=7.23, p<0.001$ \\
Stem diameter & $F_{(11,96)}=2.35, p<0.05$ & $F_{(1,96)}=0.02, p>0.05$ & $F_{(11,96)}=4.33, p<0.001$ \\
Leaf number index & $F_{(11,96)}=7.15, p<0.001$ & $F_{(1,96)}=0.41, p>0.05$ & $F_{(11,96)}=7.58, p<0.001$ \\
FRAP & $F_{(11,71)}=8.26, p<0.001$ & $F_{(1,71)}=966.60, p<0.001$ & $F_{(11,71)}=7.28, p<0.001$ \\
\hline
\end{tabular}


a
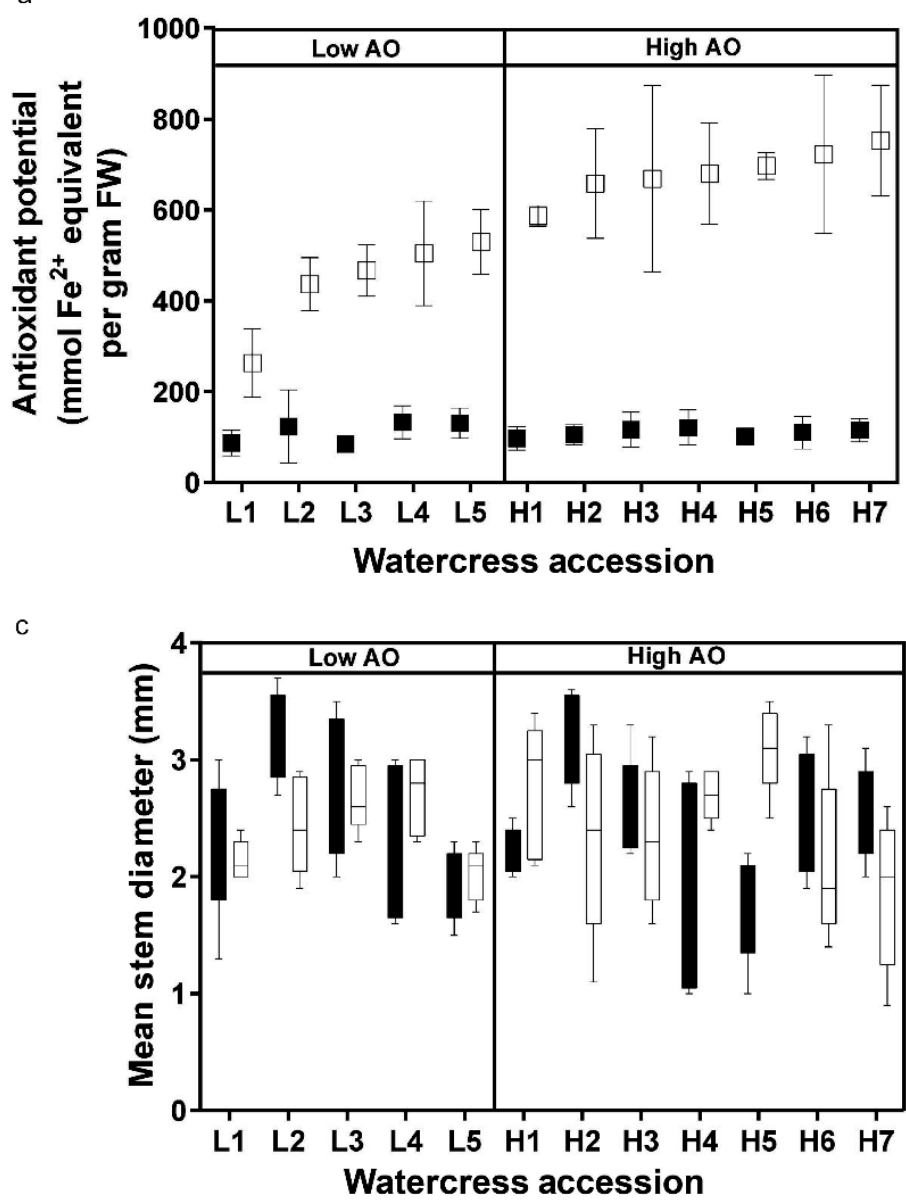

b

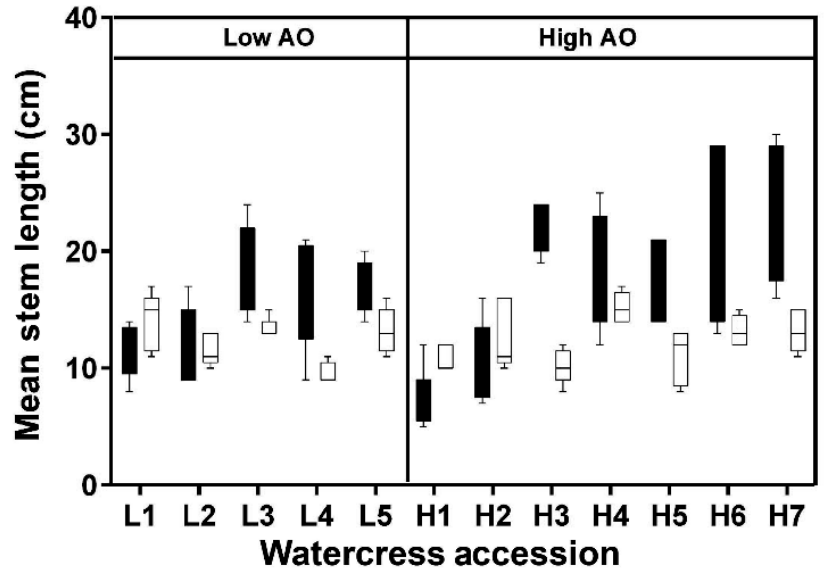

d

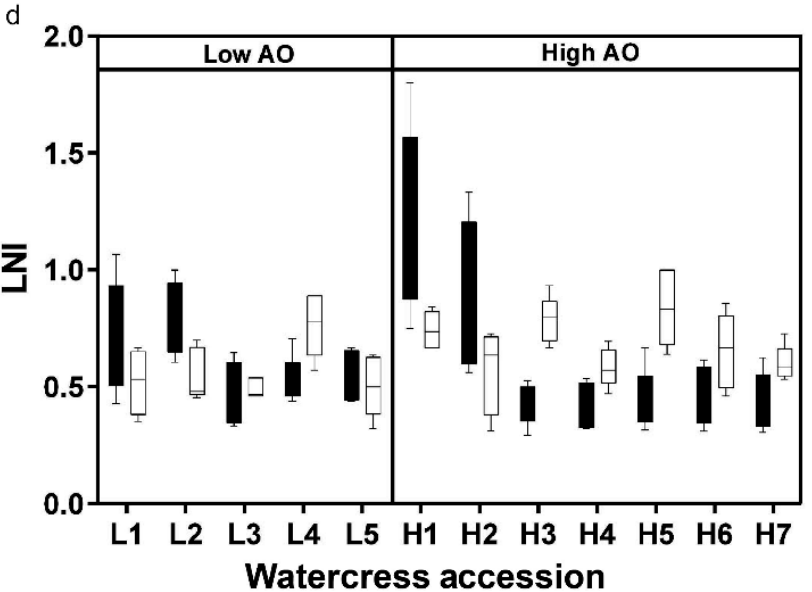

Field

\begin{tabular}{cccc}
\hline Trait & Accession & Environment & $\begin{array}{c}\text { Accession } \times \\
\text { Environment }\end{array}$ \\
Stem length & $* * *$ & $* * *$ & $* * *$ \\
Stem diameter & $*$ & ns & $* * *$ \\
Leaf number index & $* * *$ & ns & $* * *$ \\
FRAP & $* * *$ & $* * *$ & $* * *$ \\
\hline
\end{tabular}

Figure 1. (a) anti-oxidant potential (b) mean stem length (c) mean stem diameter (d) leaf number index of the watercress accessions held within the germplasm collection. L1-5 represent watercress accessions which had a low anti-oxidant potential $\left(<600 \mathrm{mmol} \mathrm{Fe}{ }^{2+}\right.$ per gram FW) whilst H1-7 represent accessions with a high anti-oxidant potential ( $>600 \mathrm{mmol} \mathrm{Fe}{ }^{2+}$ per gram FW). Asterisks identify significance from two way ANOVA analysis $* p<0.05, * * p<0.01, * * * p<0.001$. CE refers to the growth of the plants in a controlled environment in which environmental conditions are set to ensure minimum fluctuations and therefore any morphological or biochemical differences can be attributed to genes and not the environment.

(Figure 2c). This could explain some biochemical and phenotypic observations previously recorded. From the output of TMeV, seven gene clusters were revealed with two of these with significant GO term categories, i.e. enough similarity to classify the cluster with overall functions. In order to reveal the function of the genes in each of the clusters the data were uploaded to AgriGO. ${ }^{30}$ Clusters 1 and 2 had significant GO term categories where cluster 1 was mainly associated with cellular processes, cellular component organisation, cellular component biogenesis, multicellular organismal pro- cess, developmental process and response to stimulus whilst those in 2 were associated with metabolic process, cellular process and response to stimulus. Clusters 3-7 had no GO term which defined the genes in each cluster therefore although they have similar expression patterns the functions vary.

Mapman - visualisation of metabolic and cellular processes Overall there was a clear differentiation in gene expression in the dwarf phenotype in which plant defence was up-regulated whilst 
a

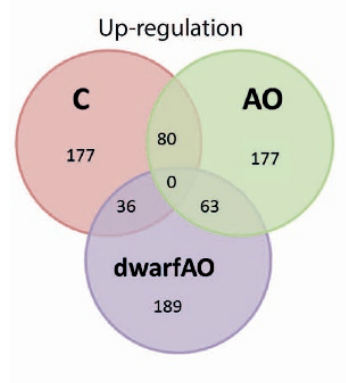

。
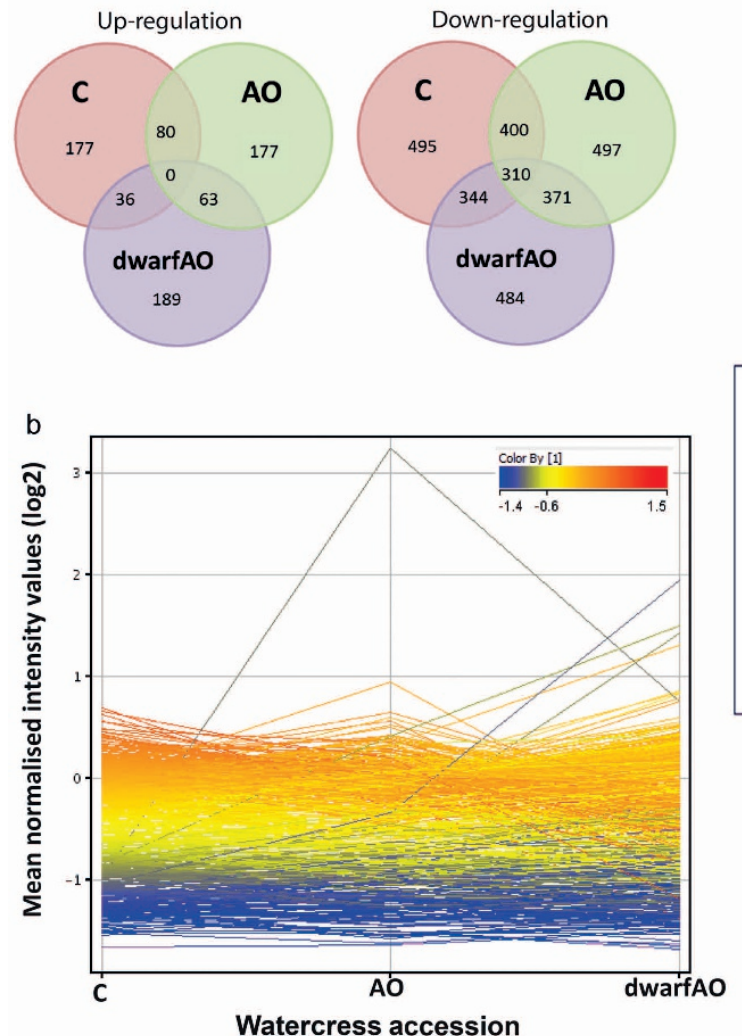

c

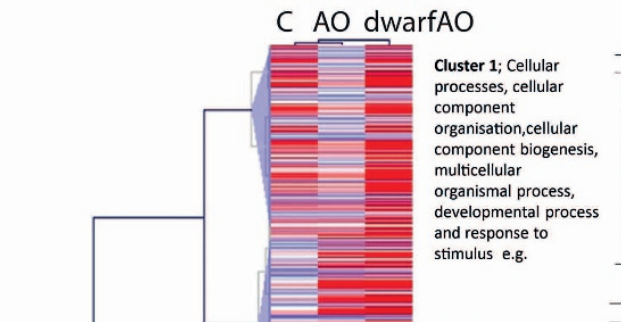

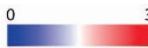

C AO dwarfAO

政

Cellular component multicellular organismal process,
developmental process and response to

Cluster 2; Metabolic process, cellular process response to
stimulus e.g. stimulus e.g.

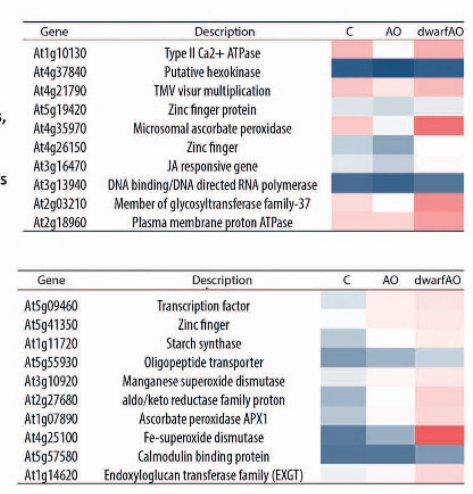

Cluster 3-7; No GO term which defined the genes in each cluster have similar expression

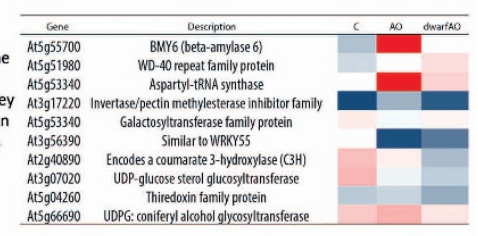

Figure 2. (a) Up- and down-regulated gene expression for control (C), high anti-oxidant (AO) and dwarf, high AO accession (dwarfAO). (b) Mean expression ratio for total gene expression of $\mathrm{C}, \mathrm{AO}$ and dwarf $\mathrm{AO}$, where green represents down-regulation, pink represents an up-regulation of genes in accession $C$ when RMA normalisation to the median was performed. This provides clear visualisation of differing expression of specific genes between three watercress accessions. For example, a gene which is down-regulated in C will have a green line despite being up- or downregulated in $\mathrm{AO}$ and dwarfAO due to the colour of the line being dependent on expression in $\mathrm{C}$. This aids the visualisation of the change in expression without having to trace the accession back to $C$. (c) Hierarchical clustering of changes in transcript abundance for $C$ and $A O$ and dwarfAO, performed using TMeV software from normalised expression ratio data. Lilac triangles represent seven different clusters, colour scale indicates signal normalised ratios (red $=$ up-regulated genes, blue $=$ down-regulated genes). Only genes with ANOVA significance $p \leqslant 0.05$ are shown.

plant growth was down-regulated (Figure 3a). This is clearly identified by examining bins in relation to plant growth and defence. dwarfAO exhibited a down-regulation of genes involved in the control of plant growth (brassinosteroids, phenylpropanoids, lignin) and an up-regulation of genes in plant defence (glucosinolates, cyanogenic glycosides, glutathione-S-transferase and ascorbate/ glutathione). Gibberellins and brassinosteroids are plant hormones which are required for growth and development. Commonly associated with a dwarf phenotype is a reduction in either of these hormones, plants which are unable to perceive brassinosteroids exhibit typical phenotypes which include the dwarf phenotype. ${ }^{31}$ The brassinosteroids have been reported to be involved in organ growth, cell expansion, cell division and responses to abiotic and biotic stresses. ${ }^{32}$ Gene expression analysis from Mapman reveals a down-regulation in the bin representing brassinosteroids for the following genes; Rotundifolia3 (at4g36380), Sterol methyltransferase2 (at1g20330), 7RED (at1g50430) and multiple hits which correspond to brassinosteroid metabolism. For example, ROTUNDIFOLIA3 encodes an enzyme to catalyse C-23 hydroxylation of several brassinosteroids which is in fact two steps shorter than the conventional brassionsteroid pathway, ${ }^{32,33}$ this gene is down-regulated in dwarfAO.

Also any deviation in lignin biosynthesis, whether that is in terms of lignin modification, can result in a dwarf phenotype or developmental arrest. ${ }^{34}$ Consistently down-regulated in dwarfAO is phenylalanine ammonia-lyase (PAL) (at2g37040) along with 4-coumarate-CoA ligase (at1g65060), UDPG:coniferyl alcohol glucosyltransferase (at5g66690) and O-methyltransferase1 (at5g54160), involved in the phenylpropanoid pathway and lignin biosynthesis. ${ }^{34,35,36,37,38}$ PAL catalyses the first step in the phenylpropanoid pathway which involves the deamination of Phe to cinnamic acid. ${ }^{39}$ A study by Huang et al. ${ }^{39}$ reported a stunted and sterile Arabidopsis with the quadruple knockout of the four PAL genes, also the levels of lignin were greatly reduced. Genes in the bin regarding the cell wall were also down-regulated. Monolignol biosynthesis mutants have dwarf phenotypes as many different disruptions in lignin biosynthesis may result in a dwarfs, however further work for confirmation is required. ${ }^{34}$ Within the watercress accessions a down-regulation of lignin is evident in dwarfAO and also a high proportion of genes are down-regulated in terms of the phenylpropanoid pathway. There is a high proportion of downregulation in the bin associated with development which may be a result of a variety of down-regulation in terms of brassinosteroids, lignin and the phenylpropanoid pathway resulting in the dwarf phenotype.

Perhaps more importantly here, is the initial step in the biosynthesis of glucosinolate - the key chemical constituents contributing to the anti-cancer properties in brassica crops including watercress and broccoli. $^{40,41,42}$ Glucosinolate biosynthesis was up-regulated in dwarfAO 


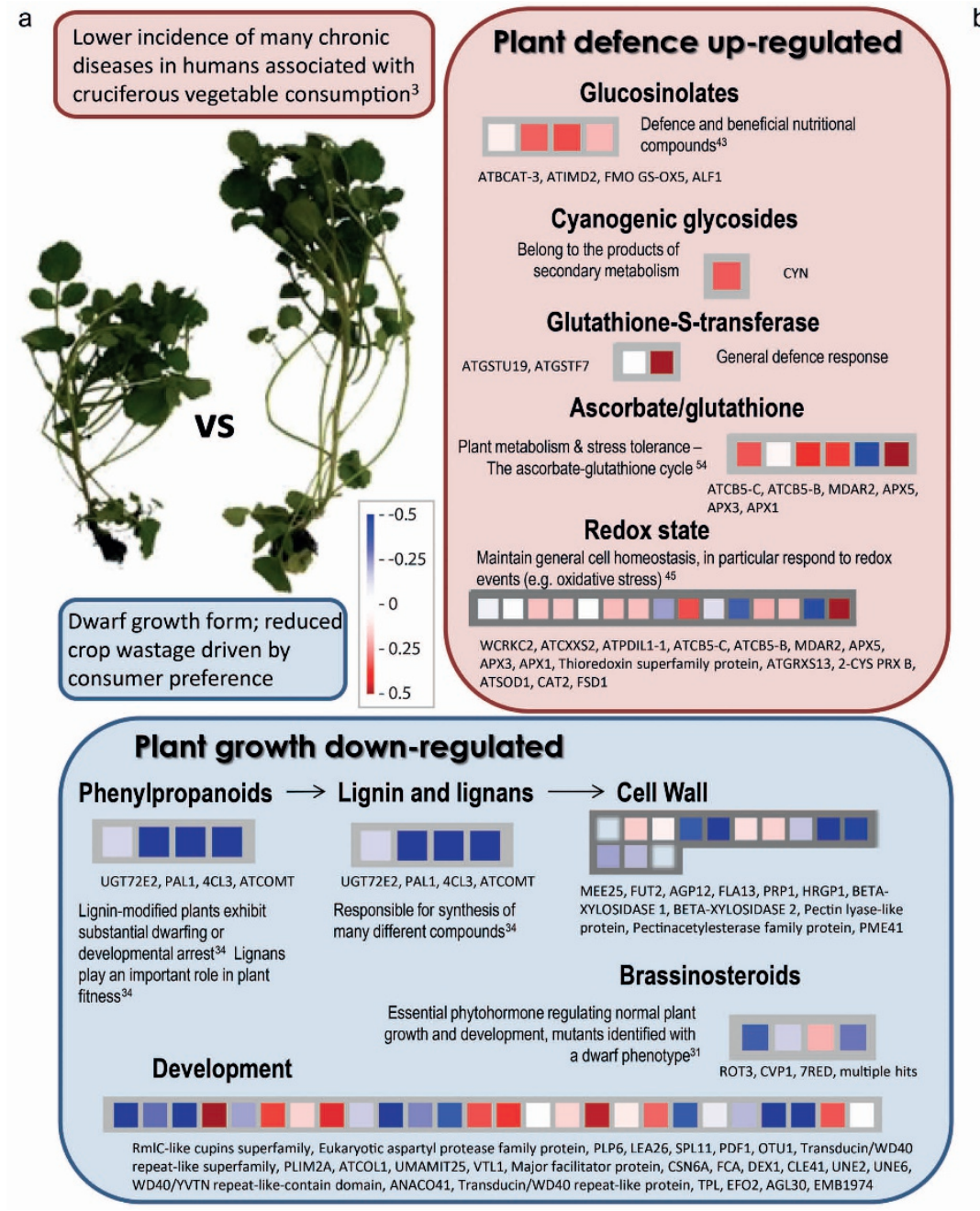

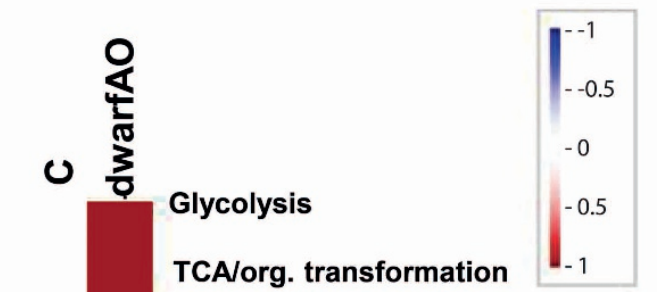
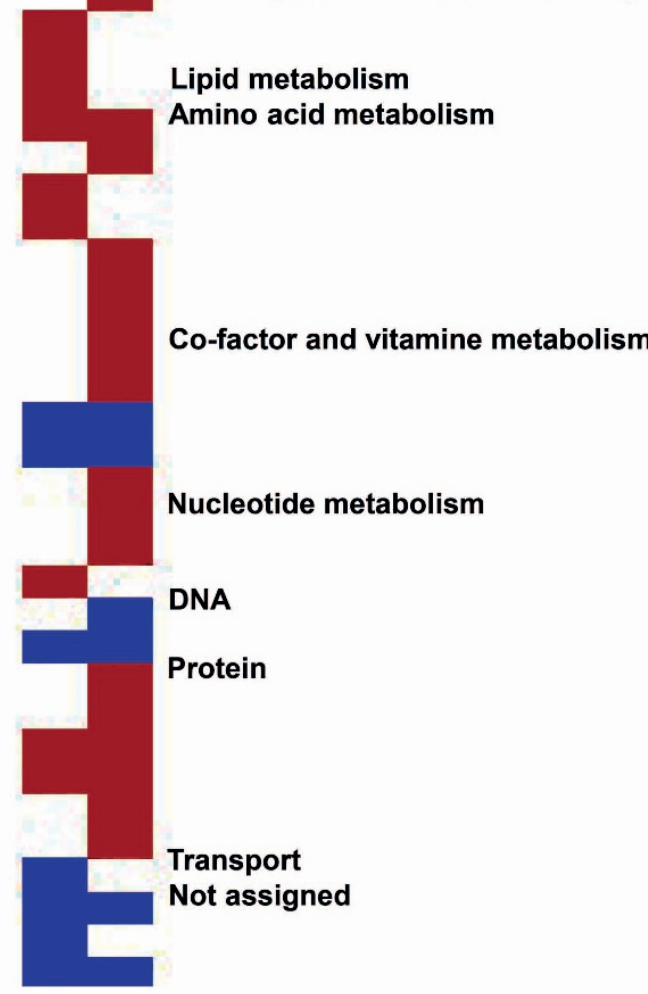

Figure 3. (a) Transcriptomic differences between a novel dwarf watercress accession (dwarfAO) and the commercial (C) accession, where an upor down- regulations, red and blue squares respectively is shown in dwarfAO compared to C. Mapman output illustrating down-regulation of genes in bins relating to plant growth in dwarfAO/C and an up-regulation of genes in the bins relating to plant defence in watercress accession dwarfAO/C. Superscript numbers denote Refs. 43, 54, 45, 34, 31, 3. (b) Overview of significantly different functional categories after microarray analysis. Colour scale represents fold changes, blue down-regulation and red up-regulation. Wilcoxon rank-sum test was applied to all three comparisons performed in PageMan. Clear visual representation of how the functional ontologies differ between the two watercress accessions.

confirming the likely improved beneficial bioactive compounds for humans in this watercress accession. ${ }^{43}$ Glucosinolates up-regulated include 3-isopropylmalate dehydrogenase (at1g80560) and a flavincontaining monooxygenase family protein (at1g12140). Ascorbate was also up-regulated which scavenges hydrogen peroxide, and is an important AO system of vital importance in combating oxidative stress, alongside an up-regulation of ascorbate peroxidise (at1g07890). Other genes which were up-regulated in the bin responsible for genes encoding ascorbate/glutathione include cytochrome b5 family protein \#1 (at2g46650) monodehydroascorbate reductase (at5g03630), cytochrome B5 isoform (at2g32720), ascorbate peroxidase 5 (at4g35970) and ascorbate peroxidase 3 (at4g35000). Glutathione is of up-most importance to plants as plants are unable to survive without glutathione. It plays a function in plant development which cannot be performed by any other thiols or AOs. ${ }^{44}$ Glutathione also plays a role in prevention of oxidative stress. Redox status takes into account all the different redox compounds including ascorbate, glutathione, $\mathrm{NAD}(\mathrm{P}) \mathrm{H}$ and proteins from the thioredoxin, ${ }^{45}$ two of which have been mentioned. Genes in the bin regarding redox status are up-regulated in order to maintain a general redox homeostasis thereby preventing oxidative stress. The down-regulation of many genes associated with plant growth and the up-regulation of genes associated with plant defence may contribute to an understanding in the phenotype and improved nutritional properties in the dwarfAO.

Pageman - visual representation of functional ontologies Gene expression data were uploaded to Pageman (Figure 3b). It is apparent that accession $\mathrm{C}$ and dwarfAO differ in the significant functional ontologies. dwarfAO has a significant up-regulation of the following functional ontologies; glycolysis, TCA/org. transformation, co-factor and vitamine metabolism, nucleotide metabolism and DNA whilst accession C lipid metabolism and transport. Functional ontologies which are significant for both accessions include amino acid metabolism and non-assigned function. This reinforces the output of the $\mathrm{TMeV}$ which was used to investigate clustering of genes. From this programme, it was evident that $C$ and $\mathrm{AO}$ were more closely related in respect to gene expression patterns than dwarfAO. This may also explain the distinctive dwarf 
phenotype and high $A O$ potential of dwarf $A O$ compared to $C$ and $A O$. Also when the gene expression analysis was examined using Venn diagrams for $\mathrm{C}$ and dwarfAO it was clear that there were fewer genes in common which were up- or down-regulated.

\section{DISCUSSION}

The watercress germplasm collection investigated here represents an important repository of genetic diversity for future breeding effort in this species. Currently, no active breeding programme exists for watercress, globally, which is surprising given the important nutrient intensity of this crop relative to others. ${ }^{1}$ This study has taken the important first steps in revealing the potential of this crop. Introgression of alleles from wild relatives of crop plants is recognised as a valuable route for yield and quality improvements. ${ }^{46}$ Tanksley and McCouch ${ }^{47}$ highlight the need for germplasm collections to unlock valuable genetic information from wild relatives. Plant breeders wish to optimise the use of natural genetic variation by bringing together favourable alleles (such as maximising yield or increased resistance to stress) in one genotype ${ }^{48}$ and this will continue to play a vital role in agriculture and has contributed to increased crop productivity. ${ }^{49}$ Watercress undoubtedly has a large number of nutritional characteristics, which have yet to be introgressed into directional breeding. This study has identified watercress accessions with favourable traits both nutritionally and agronomically which can contribute to breeding programmes.

Initial phenotypic measurements included stem length, stem diameter and LNI. These showed promising variation within the collection along with biochemical analysis (FRAP for AOs). Germplasm collections offer a valuable source of genetic variation, ${ }^{50}$ of particular interest here was variation in stem length alongside beneficial nutritional compounds that contribute to plant defence and disease-preventing phytonutrients. ${ }^{42}$ Glucosinolates are sulphur containing compounds and upon breakdown form products which have beneficial e.g. anti-cancer properties. ${ }^{40}$ It is the characteristic peppery taste of watercress; other Brassicas also have distinct flavours, which contribute to cancer prevention. ${ }^{40,42} \mathrm{~A}$ breakdown product of glucosinolates which is vital to the beneficial anti-cancer properties of watercress is phenethyl isothiocyanate (PEITC, glucosinolate derivative). PEITC can inhibit cancer development by preventing carcinogen activation by; inhibiting phase 1 enzymes, ${ }^{41}$ increasing activity of detoxifying/AO phase ॥ enzymes, ${ }^{41}$ inhibiting uncontrolled growth of cancer cells by inhibiting cell cycle progression, ${ }^{51}$ inducing apoptosis ${ }^{52}$ and anti-angiogenesis which prevents the formation of new blood vessels to a tumour. ${ }^{53}$ As stated by Cartea and Velasco, ${ }^{40}$ variation in glucosinolates can be attributed to both genetics and environment and here we reveal both a genetic and an environmental effect of glucosinolate metabolism with very clear differences AO potential revealed in the contrasting growth environments. The established germplasm collection is likely to contain valuable variability in the beneficial compounds, glucosinolates.

Transcriptome analysis of three accessions; C, AO and dwarfAO revealed variation in gene expression. In particular the expression of an ascorbate peroxidase defending against hydrogen peroxide ${ }^{54}$ and an isoform of 4-coumarate:CoA ligase (4CL) involved in the final steps of the phenylpropanoid pathway. ${ }^{55}$ There was an intriguing level of variation of gene expression between the three different watercress accessions, in particular between $\mathrm{C}$ and dwarfAO. Gene expression analysis indicated a high investment in defence chemistry by dwarfAO. For successful growth, plants are required to allocate resources between numerous processes ${ }^{56}$ and the growth-differentiation balance hypothesis predicts the allocation of resources. ${ }^{57}$ It is of great importance that the plant grows quickly in order to compete and also maintain defences in order to survive attack. ${ }^{58}$ dwarfAO is a valuable target for breeding as it appears to partition more resources into defence compared to growth, it has a dwarf, small leaved phenotype and a raised concentration of glucosinolates suggesting better disease-prevention phytonutrition.

This study has demonstrated the ability to examine the expression of thousands of genes and detect differences in gene expression using microarray analysis. Further steps can be initiated for a molecular breeding programme based on the vast array of genetic information now available from this study.

In conclusion this research provides the first analysis of the watercress transcriptome and has opened up a vast array of gene expression data for this crop; indeed, it has also highlighted genes of interest. By utilising a collection with considerable natural genetic diversity, we have also quantified significant phenotypic and biochemical differences related to yield, morphology and biochemical traits underpinning the health benefits of watercress consumption. This valuable pre-breeding data can now be extended to develop a targeted molecular breeding programme in watercress. Initial investigation into the watercress germplasm collection has provided exciting results which act as a valuable platform in which to progress further in terms of breeding for an enhanced cultivar. Current research has used the information here to develop new crosses, an RNASeq transcriptomic data set (Voutsina personal communication) and molecular genetic map of watercress interest. The impact of commercial breeding has resulted in a very narrow genetic basis for many crops, fortunately this study has revealed this may not be the case for watercress.

\section{COMPETING INTERESTS}

We confirm we have no conflict of interests.

\section{ACKNOWLEDGEMENTS}

Research on watercress in the laboratory of GT is supported by The University of Southampton and Vitacress Salads Ltd.

\section{REFERENCES}

1 Di Noia J. Defining powerhouse fruits and vegetables: a nutrient density approach. Prev Chronic Dis 2014; 11: E95.

2 Gill Cl, Haldar S, Boyd LA, Bennett R, Whiteford J, Butler M et al. Watercress supplementation in diet reduces lymphocyte DNA damage and alters blood antioxidant status in healthy adults. Am J Clin Nutr 2007; 85: 504-510.

3 Manchali S, Murthy KNC, Patil BS. Crucial facts about health benefits of popular cruciferous vegetables. J Funct Foods 2012; 4: 94-106.

4 Singh SV, Singh K. Cancer chemoprevention with dietary isothiocyanates mature for clinical translational research. Carcinogenesis 2012: 33: 1833-1842.

5 Cavell BE, Alwi SSS, Donlevy A, Packham G. Anti-angiogenic effects of dietary isothiocyanates: mechanisms of action and implications for human health. Biochem Pharmacol 2011; 81: 327-336.

6 Fimognari C, Turrini E, Ferruzzi L, Lenzi M, Hrelia P. Natural isothiocyanates: genotoxic potential versus chemoprevention. Mutat Res/Rev Mutat Res 2012; 750: 107-131.

7 Dinkova-Kostova AT, Kostov RV. Glucosinolates and isothiocyanates in health and disease. Trend Molec Med 2012; 18: 337-347.

8 Getahun SM, Chung F-L. Conversion of glucosinolates to isothiocyanates in humans after ingestion of cooked watercress. Cancer Epidemiol Biomarkers Prev 1999; 8: 447-451.

9 Martin C, Zhang Y, Tonelli C, Petroni K. Plants, diet, and health. Annu Rev Plant Biol 2013; 64: 19-46.

10 Wirsenius S, Azar C, Berndes G. How much land is needed for global food production under scenarios of dietary changes and livestock productivity increases in 2030? Agricult Syst 2010; 103: 621-638.

11 Davey JW, Hohenlohe PA, Etter PD, Boone JQ, Catchen JM, Blaxter ML. Genomewide genetic marker discovery and genotyping using next-generation sequencing. Nat Rev Genet 2011; 12: 499-510.

12 Moose SP, Mumm RH. Molecular plant breeding as the foundation for 21 st century crop improvement. Plant Physiol 2008; 147: 969-977.

13 Hajjar R, Hodgkin T. The use of wild relatives in crop improvement: a survey of developments over the last 20 years. Euphytica 2007; 156: 1-13.

14 Levin I, Lalazar A, Bar M, Schaffer AA. Non GMO fruit factories: strategies for modulating metabolic pathways in the tomato fruit. Ind Crops Prod 2004; 20: 29-36. 
15 Feuillet $C$, Langridge P, Waugh R. Cereal breeding takes a walk on the wild side. Trend Genet 2008; 24: 24-32.

16 Lu Y, Huggins P, Bar-Joseph Z. Cross species analysis of microarray expression data. Bioinformatics 2009; 25: 1476-1483.

17 Hammond JP, Broadley MR, Craigon DJ, Higgins J, Emmerson ZF, Townsend HJ et al. Using genomic DNA-based probe-selection to improve the sensitivity of highdensity oligonucleotide arrays when applied to heterologous species. Plant Methods 2005; 1: 10.

18 Hammond JP, Bowen HC, White PJ, Mills V, Pyke KA, Baker AJ et al. A comparison of the Thlaspi caerulescens and Thlaspi arvense shoot transcriptomes. New Phytol 2006; 170: 239-260.

19 Davey MW, Graham NS, Vanholme B, Swennen R, May ST, Keulemans J. Heterologous oligonucleotide microarrays for transcriptomics in a non-model species: a proof-of-concept study of drought stress in Musa. BMC Genomics 2009; 10: 436.

20 Morinaga SI, Nagano AJ, Miyazaki S, Kubo M, Demura T, Fukuda H et al. Ecogenomics of cleistogamous and chasmogamous flowering: genome-wide gene expression patterns from cross-species microarray analysis in Cardamine kokaiensis (Brassicaceae). J Ecol 2008; 96: 1086-1097.

21 Misson J, Raghothama KG, Jain A, Jouhet J, Block MA, Bligny R et al. A genome-wide transcriptional analysis using Arabidopsis thaliana Affymetrix gene chips determined plant responses to phosphate deprivation. Proc Natl Acad Sci U S A 2005; 102: 11934-11939.

22 Cavell AC, Lydiate D, Parkin I, Dean C, Trick M. Collinearity between a 30 centimorgan segment of Arabidopsis thaliana chromosome 4 and duplicated regions within the Brassica napus genome. Genome 1998; 41: 62-69.

23 Muangprom A, Osborn T. Characterization of a dwarf gene in Brassica rapa, including the identification of a candidate gene. Theor Appl Genet 2004; 108: 1378-1384.

24 Payne AC, Mazzer A, Clarkson GJ, Taylor G. Antioxidant assays - consistent findings from FRAP and ORAC reveal a negative impact of organic cultivation on antioxidant potential in spinach but not watercress or rocket leaves. Food $\mathrm{SCi}$ Nutr 2013; 1: 439-444.

25 Benzie IF, Strain J. The ferric reducing ability of plasma (FRAP) as a measure of "antioxidant power": the FRAP assay. Anal Biochem 1996; 239: 70-76.

26 Doyle JJ. A rapid DNA isolation procedure for small quantities of fresh leaf tissue. Phytochem Bull 1987; 19: 11-15.

27 Park PJ, Cao YA, Lee SY, Kim J-W, Chang MS, Hart R et al. Current issues for DNA microarrays: platform comparison, double linear amplification, and universal RNA reference. J Biotechnol 2004; 112: 225-245.

28 Pfaffl MW. A new mathematical model for relative quantification in real-time RTPCR. Nucleic Acids Res 2001; 29: e45.

29 Saeed Al, Bhagabati NK, Braisted JC, Liang W, Sharov V, Howe EA et al. TM4 microarray software suite. Methods Enzymol 2006; 411: 134-193.

30 Du Z, Zhou X, Ling Y, Zhang Z, Su Z. agriGO: a GO analysis toolkit for the agricultural community. Nucleic Acids Res 2010: 38: W64-W70.

31 Guo Z, Fujioka S, Blancaflor EB, Miao S, Gou X, Li J. TCP1 modulates brassinosteroid biosynthesis by regulating the expression of the key biosynthetic gene DWARF4 in Arabidopsis thaliana. Plant Cell 2010; 22: 1161-1173.

32 Polko JK, Pierik R, van Zanten M, Tarkowská D, Strnad M, Voesenek LA et al. Ethylene promotes hyponastic growth through interaction with ROTUNDIFOLIA3/CYP90C1 in Arabidopsis. J Exp Bot 2012: ers356.

33 Mizutani M, Ohta D. Diversification of P450 genes during land plant evolution. Annu Rev Plant Biol 2010; 61: 291-315.

34 Bonawitz ND, Chapple C. Can genetic engineering of lignin deposition be accomplished without an unacceptable yield penalty? Curr Opin Biotechnol 2013; 24: 336-343.

35 Do C-T, Pollet B, Thévenin J, Sibout R, Denoue D, Barrière $Y$ et al. Both caffeoyl Coenzyme A 3-O-methyltransferase 1 and caffeic acid O-methyltransferase 1 are involved in redundant functions for lignin, flavonoids and sinapoyl malate biosynthesis in Arabidopsis. Planta 2007; 226: 1117-1129.

36 Im Kim J, Ciesielski PN, Donohoe BS, Chapple C, Li X. Chemically-induced conditional rescue of the ref8 mutant of Arabidopsis thaliana reveals rapid restoration of growth and selective turnover of secondary metabolite pools. Plant Physiol 2013: 164: 584-595.

37 Neutelings G. Lignin variability in plant cell walls: contribution of new models. Plant Sci 2011; 181: 379-386.

38 Schilmiller AL, Stout J, Weng JK, Humphreys J, Ruegger MO, Chapple C. Mutations in the cinnamate 4-hydroxylase gene impact metabolism, growth and development in Arabidopsis. Plant J 2009; 60: 771-782.

39 Huang J, Gu M, Lai Z, Fan B, Shi K, Zhou Y-H et al. Functional analysis of the Arabidopsis PAL gene family in plant growth, development, and response to environmental stress. Plant Physiol 2010; 153: 1526-1538.

40 Cartea M, Velasco P. Glucosinolates in Brassica foods: bioavailability in food and significance for human health. Phytochem Rev 2008; 7: 213-229.

41 Rose P, Faulkner K, Williamson G, Mithen R. 7-Methylsulfinylheptyl and 8methylsulfinyloctyl isothiocyanates from watercress are potent inducers of phase II enzymes. Carcinogenesis 2000; 21: 1983-1988.

42 Herr I, Büchler MW. Dietary constituents of broccoli and other cruciferous vegetables: implications for prevention and therapy of cancer. Cancer Treat Rev 2010; 36: 377-383.

43 Baskar V, Gururani MA, Yu JW, Park SW. Engineering glucosinolates in plants: current knowledge and potential uses. Appl Biochem Biotechnol 2012; 168: 1694-1717.

44 Noctor G, Mhamdi A, Chaouch S, Han Y, Neukermans J, Marquez-Garcia B et al. Glutathione in plants: an integrated overview. Plant Cell Environ 2012; 35: 454-484.

45 Potters G, Horemans N, Jansen MA. The cellular redox state in plant stress biology a charging concept. Plant Physiol Biochem 2010; 48: 292-300.

46 Lin T, Zhu G, Zhang J, Xu X, Yu Q, Zheng Z et al. Genomic analyses provide insights into the history of tomato breeding. Nat Genet 2014; 46: 1220-1226.

47 Tanksley SD, McCouch SR. Seed banks and molecular maps: unlocking genetic potential from the wild. Science 1997; 277: 1063-1066.

48 Koornneef M, Stam P. Changing paradigms in plant breeding. Plant Physiol 2001 125: $156-159$.

49 Lee M. Genome projects and gene pools: new germplasm for plant breeding? Proc Natl Acad Sci U S A 1998; 95: 2001-2004.

50 Virk PS, Ford-Lloyd BV, Jackson MT, Newbury HJ. Use of RAPD for the study of diversity within plant germplasm collections. Heredity 1995; 74: 170-179.

51 Chiao JW, Wu H, Ramaswamy G, Conaway CC, Chung F-L, Wang L et al. Ingestion of an isothiocyanate metabolite from cruciferous vegetables inhibits growth of human prostate cancer cell xenografts by apoptosis and cell cycle arrest. Carcinogenesis 2004; 25: 1403-1408.

52 Satyan K, Swamy N, Dizon DS, Singh R, Granai CO, Brard L. Phenethyl isothiocyanate (PEITC) inhibits growth of ovarian cancer cells by inducing apoptosis: role of caspase and MAPK activation. Gynecol Oncol 2006; 103: 261-270.

53 Kenfield SA, Chang ST, Chan JM. Diet and lifestyle interventions in active surveillance patients with favorable-risk prostate cancer. Curr Treat Options Oncol 2007; 8: 173-196.

54 Noctor G, Foyer $\mathrm{CH}$. Ascorbate and glutathione: keeping active oxygen under control. Ann Rev Plant Biol 1998; 49: 249-279.

55 Ferrer J-L, Austin M, Stewart Jr C, Noel J. Structure and function of enzymes involved in the biosynthesis of phenylpropanoids. Plant Physiol Biochem 2008; 46: 356-370.

56 Cronin G, Hay ME. Effects of light and nutrient availability on the growth, secondary chemistry, and resistance to herbivory of two brown seaweeds. Oikos 1996; 73: 93 106.

57 Stamp N. Can the growth-differentiation balance hypothesis be tested rigorously? Oikos 2004; 107: 439-448.

58 Herms DA, Mattson WJ. The dilemma of plants: to grow or defend. Quart Rev Biol 1992: 283-335.95.

(c) (i) This license allows readers to copy, distribute and transmit the Contribution as long as it is attributed back to the author. Readers are permitted to alter, transform or build upon the Contribution, and to use the article for commercial purposes. Please read the full license for further details at - http:// creativecommons.org/licenses/by/4.0/

Supplementary information accompanies the article can be found on the Horticulture Research's website (http://www.nature.com/hortres). 